\title{
UAV-Based Ground Penetrating Synthetic Aperture Radar
}

\author{
Markus Schartel, Ralf Burr, Winfried Mayer, Nando Docci, and Christian Waldschmidt
}

(C) 2018 IEEE. Personal use of this material is permitted. Permission from IEEE must be obtained for all other uses, in any current or future media, including reprinting/republishing this material for advertising or promotional purposes, creating new collective works, for resale or redistribution to servers or lists, or reuse of any copyrighted component of this work in other works.

DOI: 10.1109/ICMIM.2018.8443503 


\title{
UAV-Based Ground Penetrating Synthetic Aperture Radar
}

\author{
Markus Schartel*, Ralf Burr ${ }^{\dagger}$, Winfried Mayer ${ }^{\ddagger}$, Nando Docci ${ }^{\S}$, and Christian Waldschmidt* \\ *Institute of Microwave Engineering, Ulm University, 89081 Ulm, Germany \\ †Ulm University of Applied Sciences, 89075 Ulm, Germany \\ $\ddagger$ Endress+Hauser GmbH+Co. KG, 79689 Maulburg, Germany \\ $\S$ University of Applied Sciences and Arts Northwestern Switzerland \\ Email: markus.schartel@uni-ulm.de
}

\begin{abstract}
A novel approach for anti-personnel landmine detection using an unmanned aerial vehicle (UAV) in combination with a ground penetrating synthetic aperture radar (GPSAR) is presented. The objective of the system is to accelerate the process of land release in humanitarian demining. Suspicious objects shall be detected by the radar and marked for further investigations using different sensor principles.

The ground penetrating radar (GPR) module consists of a $1 \mathrm{GHz}$ to $4 \mathrm{GHz}$ side-looking frequency modulated continuous wave (FMCW) radar, a radar and lidar altimeter, and a real time kinematic global navigation satellite system (RTK GNSS). The image processing is done offline using a back-projection algorithm. In the theoretical part of this paper the system partitioning, the sensor module, and the position accuracy requirements are briefly described. In the experimental part of this paper synthetic aperture radar (SAR) measurements are presented.
\end{abstract}

\section{INTRODUCTION}

Anti-personnel landmines are often used in conflicts to prevent the advance of the enemy or to block humanitarian access [1]. After the hostilities, mines are a hidden and permanent threat to the returning civilians and can injure and kill people still decades later. In 2015, the landmine monitor [2] recorded 6.461 mine or ERM (explosive remnants of war) casualties.

For more than 20 years ground penetrating radars have been used to detect buried objects [1]. In comparison to metal detectors the detection capabilities of radars are not limited to metal objects only. GPRs are a promising approach to detect modern minimum metal mines. Presently, most GPR sensors for mine detection used in humanitarian demining are handheld devices which operate very close to the surface.

To accelerate the process of land release, the focus is on the technical survey. Different sensor modules shall be mounted on a low-altitude flying UAV to detect hazard-free the presence and approximate location of anti-personnel landmines.

In this paper the fundamental system concept with respect to a sensor module consisting of a ground penetrating synthetic aperture radar is presented. The task of the GPSAR is to locate and mark suspicious objects for further investigations.

The paper is organized as follows: Section II briefly describes the system partitioning. The GPSAR module is presented in Section III. In Section IV first SAR measurements are discussed, and Section $\mathrm{V}$ gives a short conclusion.

\section{SyStem PARTITIONING}

For a reliable mine detection it is beneficial to use different sensor principles. Since the payload of a UAV is limited, the overall system is separated into a carrier platform and independently operating sensor modules. Each of these modules uses a different sensor principle, e.g. radar, metal detector, infrared/hyperspectral methods, or biological/chemical methods. Depending on the application and the ambient conditions the carrier platform and/or the sensor module can be exchanged.

Since each sensor principle has its specific demands regarding flight planning, the carrier platform needs to be a rotary wing aircraft such as a helicopter or multicopter. Thus, the carrier platform can follow a trajectory or hover over certain spots.

\section{THE GPSAR SENSOR MOdUle}

The penetration depth of electromagnetic waves depends on the frequency and the dielectric properties [1]. In [3], [4] a frequency range from $0.5 \mathrm{GHz}$ to $5 \mathrm{GHz}$ is proposed for the detection of anti-personnel landmines up to a depth of $20 \mathrm{~cm}$. As a compromise between penetration depth, resolution, and geometrical dimensions suitable for UAV applications, a frequency range of $1 \mathrm{GHz}$ to $4 \mathrm{GHz}$ was selected.

The radar prototype is a single-input single-output (SISO) FMCW radar system in a bistatic configuration. A voltage controlled oscillator generates a linear frequency ramp from $1 \mathrm{GHz}$ to $2 \mathrm{GHz}$. This ramp can be doubled by a frequency multiplier resulting in a second ramp from $2 \mathrm{GHz}$ to $4 \mathrm{GHz}$. These two separate ramps are later joined in the signal processing step.

To achieve a large area throughput and to observe the payload restriction of the UAV, the GPR is operated as SAR. Hereby, the proper motion of a side-looking radar is used to scan the area under investigation. With comparatively small apertures a fine resolution in flight direction can be achieved. The SAR principle is illustrated in Fig. 1.

The UAV performs a linear flight in $x$-direction (crossrange), whereby the side-looking radar is pointing downwards at a depression angle $\alpha$ in $y$-direction (ground-range). The distance between the radar and the area under investigation is called slant range $R$. In this applicaton the altitude $H$ of the UAV is approximately between $1.5 \mathrm{~m}$ and $2.5 \mathrm{~m}$ above 


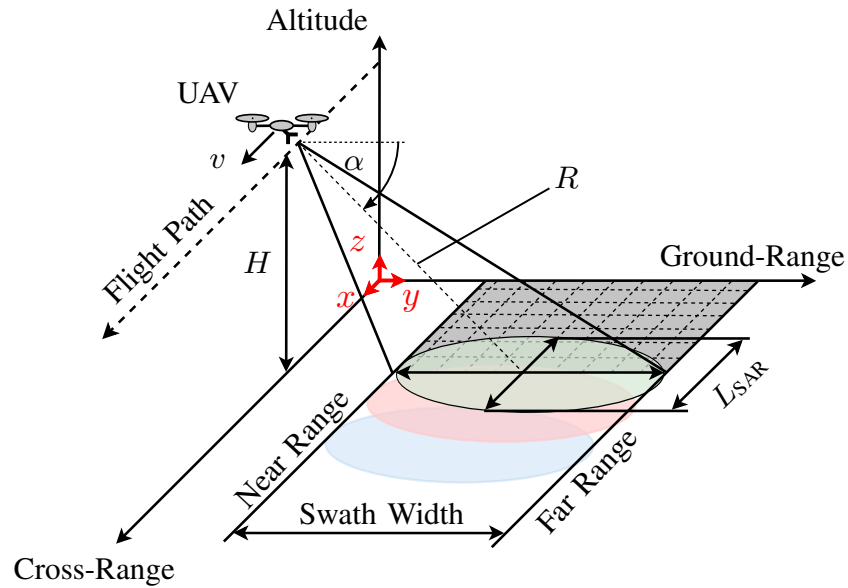

Fig. 1. Illustration of the stripmap SAR geometry.

ground. According to the antenna theory the distance between the measurements $\Delta x$ should not exceed $\frac{\lambda_{0}}{2}$ to avoid grating lobes, where $\lambda_{0}$ is the free-space wavelength. The resolution in ground-range $\delta_{\mathrm{gr}}$ is determined by the bandwidth $B$, the depression angle $\alpha$, and the beam width of the antenna in elevation $\theta_{e}$ [5]:

$$
\delta_{\mathrm{gr}}=\frac{c_{0}}{2 B \cos \left(\alpha \pm \frac{\theta_{e}}{2}\right)},
$$

where $c_{0}$ is the speed of light. Assuming a beam width $\theta_{e}=40^{\circ}$ and a depression angle $\alpha=45^{\circ}$, the ground-range resolution is between $10 \mathrm{~cm}$ in the near and $5.5 \mathrm{~cm}$ in the far range. The usable swath width is approximately $2.5 \mathrm{~m}$.

Due to the low flight altitude the resolution in cross-range $\delta_{\text {cr }}$ depends on the slant range $R[6]$ :

$$
\delta_{\mathrm{cr}}=\frac{\lambda_{0}}{4} \sqrt{1+\frac{4 R^{2}}{L_{\mathrm{SAR}}^{2}}},
$$

where $L_{\mathrm{SAR}}$ is the length of the synthetic aperture. The resolution in cross-range is between $8 \mathrm{~cm}$ in the near range and $11 \mathrm{~cm}$ in the far range.

The SAR processor has to handle low-frequency ultra wideband (UWB) FMCW radar data obtained from a nonlinear flight path in the near and far field of a wide-beamwidth antenna. Regarding frequency domain and time domain algorithms, back-projection may be the most suitable algorithm to fulfil all the demands at the expense of computing time [7][9]. The major advantages of this algorithm are the high image quality and the integrated motion compensation.

The challenge of this approach is to obtain a good 3D position accuracy of the phase center of the antenna. The green dashed curve (b) in Fig. 2 shows a sinusoidal deviation in $y$-direction from the ideal flight path (red solid (a)) with an amplitude of $\pm 50 \mathrm{~mm}$. This deviation is known. The blue dotted curve (c) shows an unknown normally distributed deviation of the ideal flight path with an amplitude of $\pm 10 \mathrm{~mm}$. The corresponding radar data and the processed SAR images are shown in Fig. 3. As long as the motion of the UAV is precisely known, the back-projection algorithm can compensate

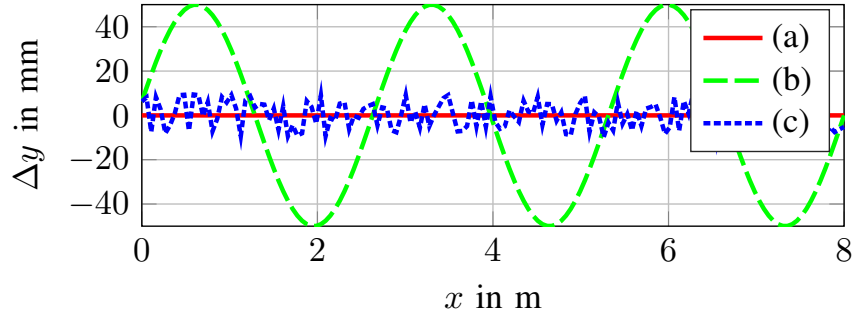

Fig. 2. Simulation of different flight paths of the UAV. (a) Ideal, (b) sinusoidal, known deviation, and (c) normally distributed, unknown deviation.
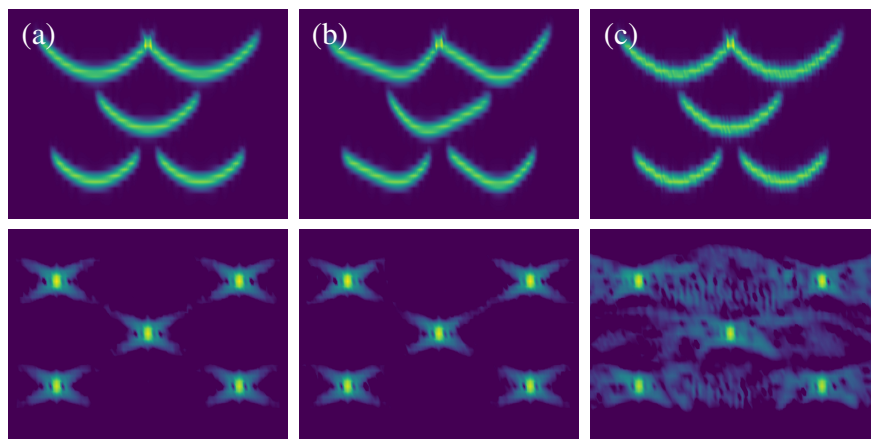

Fig. 3. Range compressed radar (upper row) and processed SAR image (lower row) for (a) ideal, (b) sinusoidal, known deviation, and (c) normally distributed, unknown deviation according to Fig. 2.

deviations of the ideal flight path. However, unknown position errors in the range of $\pm 10 \mathrm{~mm}$ influence the image quality.

The GPSAR module is shown in Fig. 4. It consists of the bistatic GPR equipped with lightweight 3D-printed horn antennas, a radar and a lidar altimeter, and a data logger. The module including a battery weights $1.6 \mathrm{~kg}$ and is mounted below the UAV.

\section{UAV-BASED SAR MEASUREMENTS}

The measurement system is shown in Fig. 6. As carrier platform a commercial available multicopter [10] was used. The selected UAV is factory fitted with 3 global satellite systems (GPS) and 3 inertial measurement units (IMUs). Additionally, for highly accurate localization the multicopter

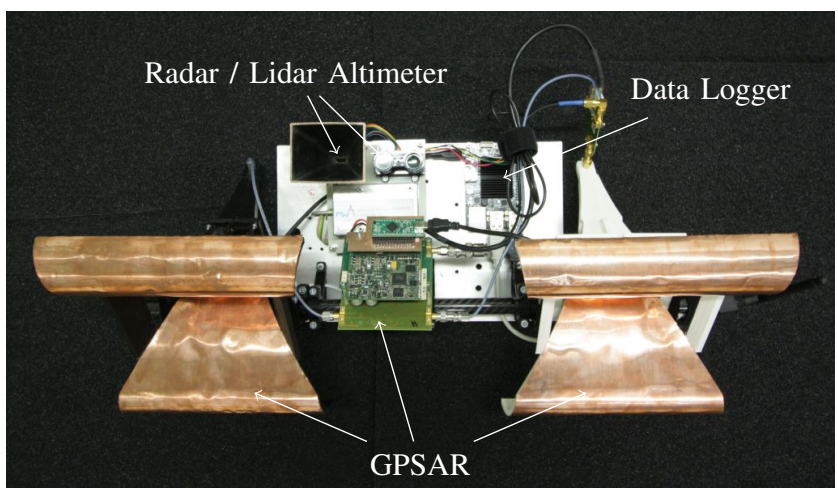

Fig. 4. Photo of the GPSAR sensor module consisting of a bistatic $1 \mathrm{GHz}$ to $4 \mathrm{GHz}$ FMCW radar, a $26 \mathrm{GHz}$ radar and lidar altimeter, and a data logger. 


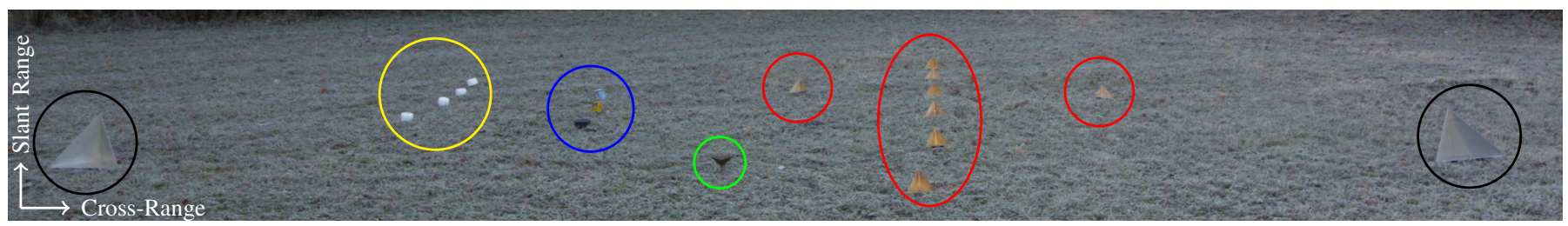

Fig. 5. Photo of the measurement setup from the perspective of the radar. The setup consists of 8 reflectors with an RCS $\sigma_{1}=-12.2 \mathrm{dBsm}$ (red), 1 reflector with an RCS $\sigma_{2}=-15.3 \mathrm{dBsm}$ pointing upwards (green), 2 corner reflectors with an RCS $\sigma_{3}=3.7 \mathrm{dBsm}$ (black), 3 metal cans (blue), and 4 plastic boxes (yellow). All targets have been placed on the ground.

was equipped with an RTK GNSS [11]. The RTK-GNSS data as well as the entire telemetry data of the UAV flight controller can be accessed via USB and is recorded by the data logger.

Despite the fact that state-of-the-art RTK-GNSS receivers promise centimeter-level accuracy, these receivers usually provide position data ( 3 degrees of freedom) only, but not the orientation. Thus, to extrapolate the exact position of the radar antenna at the time of each radar measurement, the RTKGNSS data was interpolated and has been offset with the filtered rotation data of the UAV flight controller. The data flow is illustrated in Fig. 7.

As proof of concept a stripmap SAR measurement was performed. Hereby the UAV was manually flown in an altitude of approximately $2.7 \mathrm{~m}$ parallel to the measurement setup shown in Fig. 5. As targets different sized corner reflectors, metal cans, and plastic boxes filled with beeswax have been used. All targets have been placed on the ground.

The corresponding range compressed radar data $(2 \mathrm{GHz}$ to $4 \mathrm{GHz}$ ) is shown in Fig. 8. The free space loss is not compensated. A small crosstalk between the horn antennas up to a range of $2 \mathrm{~m}$ can be observed. The first reflections from the ground are in a range of approximately $2.7 \mathrm{~m}$. The transition between free space and the first ground reflection can be used to determine the flight altitude.

The hyperbolas with vertices at $(\# 150 / 3.8 \mathrm{~m})$ and (\# 400/3.8 m) are the reflections of the corner reflectors with an RCS $\sigma_{3}=3.7 \mathrm{dBsm}$ (black circled), marking the left and right edge of the measuring field. The hyperbolas of the plastic

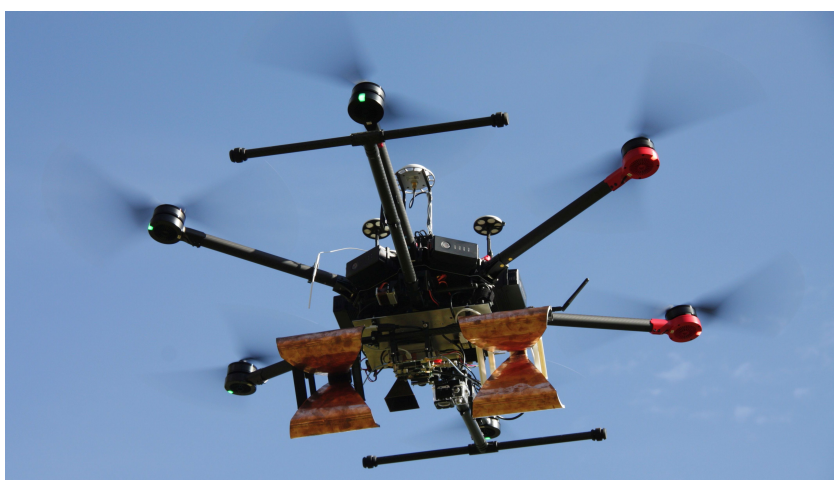

Fig. 6. Photo of the measurement system. It consists of a DJI Matrice 600 Pro, a DJI D-RTK GNSS mounted on the top of the UAV, and the GPSAR sensor module mounted below the UAV.

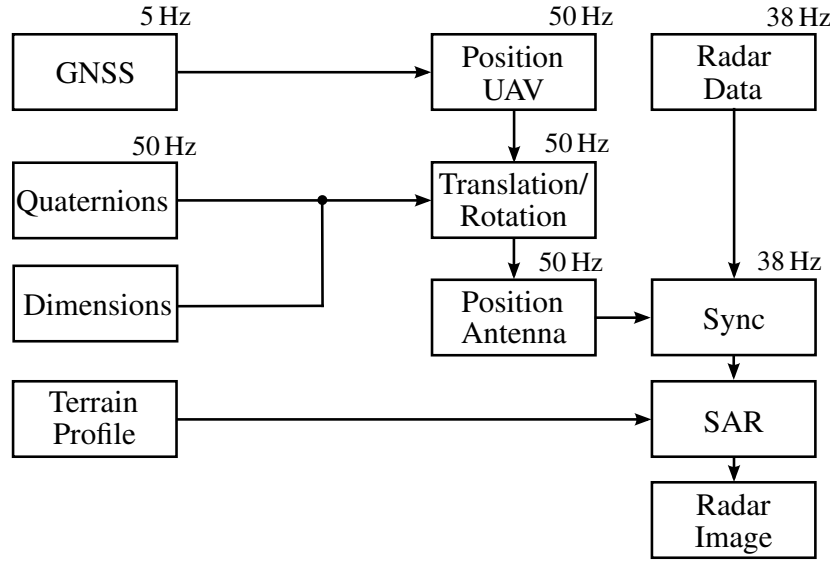

Fig. 7. Block diagram to illustrate the data flow of the processing toolchain.

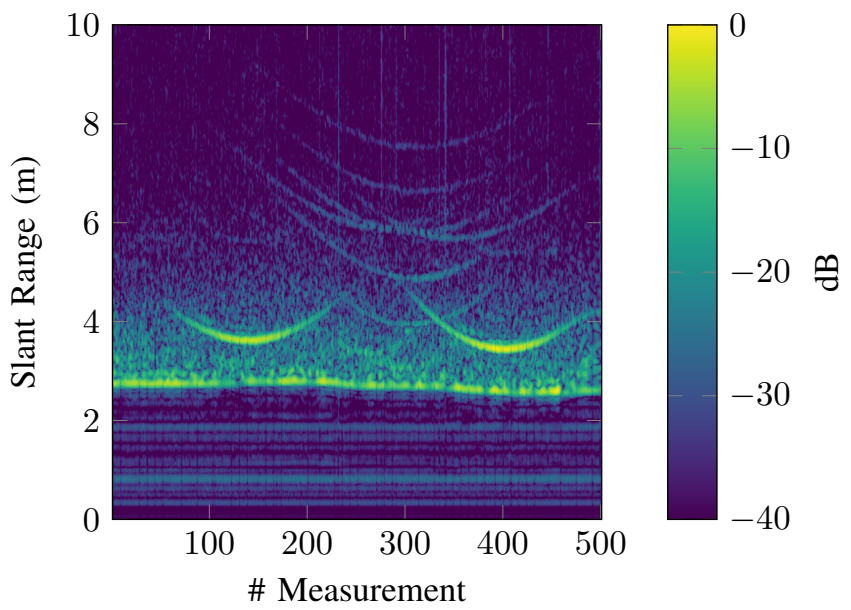

Fig. 8. Range compressed radar data ( $2 \mathrm{GHz}$ to $4 \mathrm{GHz})$.

boxes and the metal cans are not visible.

Due to accelerations of the manually steered UAV, the velocity during the stripmap SAR measurement is not constant. Since the chirp repetition frequency of the radar is fixed, the swath is not sampled equidistantly. Fulfilling the sampling theorem, the back-projection algorithm could process the data. However, areas which are illuminated a longer period of time would be weighted more, resulting in a misrepresentation of the signal strength. To compensate for this effect the radar data is weighted by the distance traversed between two 


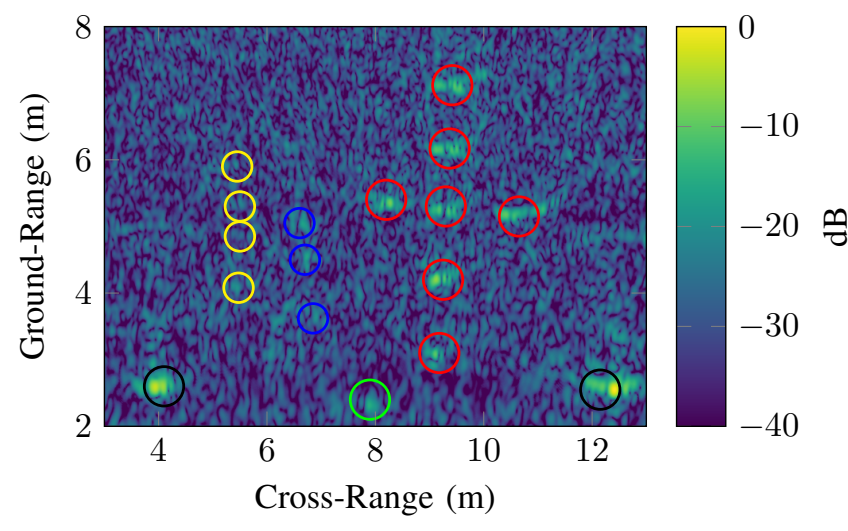

(a) GPS fused data as input data.

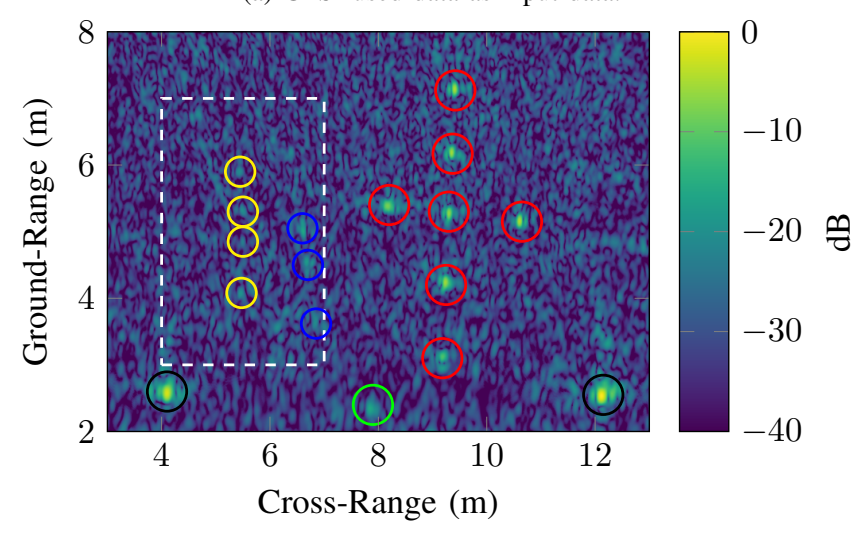

(b) RTK-GNSS data as input data.

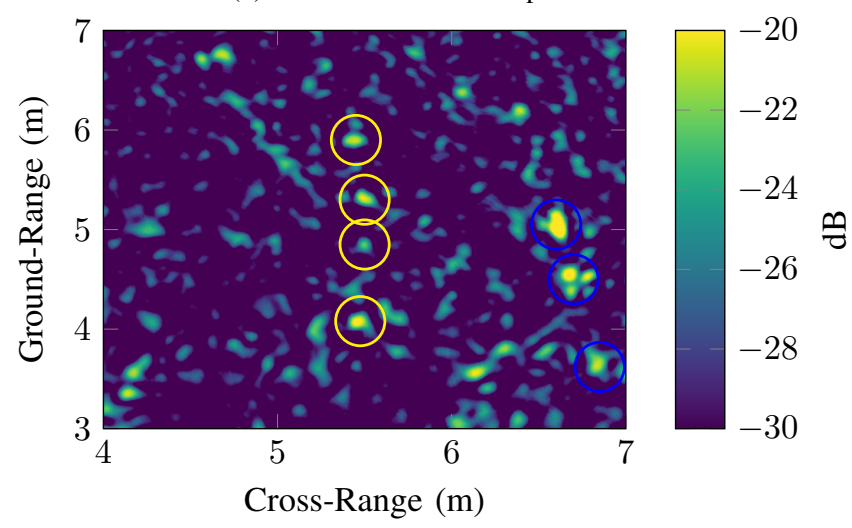

(c) Section of the image shown in Fig. 9b with adjusted dynamics.

Fig. 9. Measurement results.

measurements.

The processed SAR images for 2 different position input datasets are shown in Fig. 9. Therefore, the terrain was modelled as plane surface.

The result in Fig. 9a shows, that the position accuracy of the GPS fused data is not sufficient. The target response is blurred in cross-range direction. In addition, the location of the black circled reflectors is shifted in comparison to Fig. 9b.

If the high-precision RTK-GNSS data is used, all the reflectors can be focused very well. Even the plastic boxes can be mapped as shown in the image section in Fig. 9c. The 8th red circled reflector cannot be displayed in these images because it is located at a ground range distance of less than $2 \mathrm{~m}$.

To increase the ability to detect weak targets like plastic boxes, the position-dependent clutter needs to be suppressed e.g. by using a multiple-input multiple-output (MIMO) system [12] or by superposition of images of the same scenario recorded from different viewing angles gathered by a SISO system.

\section{CONCLUSION}

A ground penetrating synthetic aperture radar system for UAV-based mine detection has been presented. The system consists of a 1-4 GHz UWB FMCW radar, lightweight horn antennas, a radar and lidar altimeter, a data logger, an RTK GNSS, and a consumer UAV. The measurement results successfully proof the functionality of the system and show that the position accuracy of an RTK GNSS is sufficient for $1-4 \mathrm{GHz}$ stripmap SAR.

\section{ACKNOWLEDGEMENT}

The authors would like to thank the Urs Endress foundation for their support and the collaboration.

\section{REFERENCES}

[1] Daniels D. J., "Ground Penetrating Radar", 2nd Edition, Institution of Electrical Engineers, 2004

[2] International Campaign to Ban Landmines, "Landmine Monitor 2016", Monitoring and Research Committee, ICBL-CMC Governance Board, November 2016.

[3] Fischer C. and Wiesbeck W., "Multistatic GPR for antipersonnel mine detection,", IGARSS, Sydney, 2001.

[4] Fritzsche, M., "Anwendungen von Verfahren der Mustererkennung zur Detektion von Landminen mit Georadaren", Dissertation, University of Karlsruhe, 2001

[5] Klausing, H. and Holpp, W., "Radar mit realer und synthetischer Apertur: Konzeption und Realisierung", Oldenburg, 2000.

[6] Schreiber E., Peichl M., Dill S., Anger S., Heinzel A., Bischeltsrieder F., Kempf T., and Jirousek M., "Theoretical and experimental investigations of a ground-based high-resolution SAR for buried object detection," European Radar Conference (EuRAD), Paris, 2015.

[7] Peichl M., Schreiber E., Heinzel A., and Kempf T., "TIRAMI-SAR a synthetic aperture radar approach for efficient detection of landmines and UXO", Proc. of EUSAR - 10th European Conference of Synthetic Aperture Radar, March 2014.

[8] Zaugg E. C. and Long D. G., "Generalized Frequency Scaling and Backprojection for LFM-CW SAR Processing", IEEE Trans. Geoscience and Remote Sensing, July 2015

[9] Vu V. T., Sjogren T. K., and Pettersson M. I., "A Comparison between Fast Factorized Backprojection and Frequency-Domain Algorithms in UWB Lowfrequency SAR", IGARSS IEEE International Geoscience and Remote Sensing Symposium, Boston, pp. 1284-1287, 2008.

[10] DJI, "Matrice 600 Pro User Manual v1.0", 2017.

[11] DJI, "DJI D-RTK Release Notes ", 2017.

[12] Schreiber E., Peichl M., Dill S., and Bischeltsrieder F., "Detection of landmines and UXO using advanced synthetic aperture radar technology", Proc. SPIE 9823, Detection and Sensing of Mines, Explosive Objects, and Obscured Targets XXI, May 2016. 